\title{
NUMERICAL STUDIES OF THE THERMAL DESIGN SENSITIVITY \\ CALCULATION FOR A REACTION-DIFFUSION SYSTEM WITH DISCONTINUOUS DERIVATIVES ${ }^{*}$
}

\author{
Jean W. Hou and Jeen S. Sheen \\ Department of Mechanical Engineering and Mechanics \\ old Dominion University \\ Norfolk, VA
}

\section{SUMMARY}

The aim of this study is to find a reliable numerical algorithm to calculate thermal design sensitivities of a transient problem with discontinuous derivatives. The thermal system of interest is a transient heat conduction problem related to the curing process of a composite laminate. A logical function which can smoothly approximate the discontinuity is introduced to modify the system equation. Two commonly used methods, the adjoint variable method and the direct differentiation method, are then applied to find the design derivatives of the modified system. The comparisons of numerical results obtained by these two methods demonstrate that the direct differentiation method is a better choice to be used in calculating thermal design sensitivity.

\section{INTRODUCTION}

High-performance polymeric composites have been used widely in the aerospace and automobile industries. Such materials are commonly composed of long or chopped fibers embedded in the thermosetting resin matrix. Changes in physical and chemical properties of such composite materlals during the curing process are rather complex. Thus, 1 t is not a trivial task to properly design a cure cycle (temperature and pressure profiles) for a curing process. The material should be cured uniformly and completely with the lowest void content; the temperature inside the laminate must not exceed some maximum value; and the curing process should be completed within the shortest amount of time. In the past, most cure cycle designs for newly developed composite systems are based upon the technique of trial and error. Several simulation models [1-3] have been developed recently for curing various epoxy matrix composites. This development represents a significant advancement in computerizing the cure cycle design. An attempt [4] has been made recently to incorporate thermal optimal design techniques with such analysis capabilities to systematically establish the "best" curing process. The research progress regarding the computational aspects of the thermal design sensitivity analysis is reported in this paper.

* The research reported here is sponsored by NASA Langley Research Center under NASA Grant NAG-1-561. 
The derivative of the thermal response with respect to the design variable is usually called the thermal design derivative or sensitivity. The information of the design derivative is not only very useful for the trade-off design, but it is also required for an iterative design optimization. The calculation of design derivatives in thermal problems has attracted research interests in such areas as design of space structures subject to temperature constraints [5], and chemical process control $[6,7]$. The thermal system studied in this paper can be $s$ tated as a heat conduction problem coupled with chemical-kinetic reaction during the cure process, while the temperature of cure cycle is considered as a design variable.

\section{MATHEMATICAL FORMULATION OF CURING PROCESS}

During the curing process, the temperature distribution $T(x, t)$ and the degree of cure $\alpha(x, t)$ of the resin inside the composite depend on the rate at which heat is transmitted from the environment into the material. The heat conduction model for a piled composite with its thickness 2 h during the curing process can be found as

$$
\rho c \frac{\partial T}{\partial t}=k \frac{\partial^{2} T}{\partial x^{2}}+\rho H_{R} \dot{\alpha}
$$

with the boundary conditions,

$$
\begin{array}{ll}
\frac{\partial T(0, t)}{\partial x}=0 & 0<t \leqslant T \\
T(h, t)=T_{c}(t), & 0<t \leqslant T
\end{array}
$$

and the initial condition

$$
\mathrm{T}(\mathrm{x}, 0)=\mathrm{T}_{\mathrm{o}}(\mathrm{x}), \quad 0<\mathrm{x}<\mathrm{h},
$$

where $\rho$ is the mass density, $c$ is the coefficient of heat capacity, $k$ is the heat conduction coefficient, and $H_{R}$ is the total or ultimate heat of reaction during the curing process. The last term in equation (1), $\mathrm{\rho H}_{\mathrm{R}} \dot{\alpha}$, denotes the rate of heat generated by chemical reaction which can be expressed by cure kinetics.

Two models of cure kinetics are investigated here. One is the chemical-kinetic reaction of Hercules 3501 during press processing [1]. The chemical-kinetic reaction can be determined in terms of the degree of cure, $\alpha$, which is given experimentally from reference [2] as

$$
\begin{aligned}
\mathrm{f}_{1}(\alpha, \mathrm{T}, \mathrm{t}) & =\left(\mathrm{K}_{1}+\mathrm{K}_{2} \alpha\right)(1-\alpha)(\mathrm{B}-\alpha), & 0<\alpha<0.3 \\
\mathrm{f}_{2}(\alpha, \mathrm{T}, \mathrm{t}) & =\mathrm{K}_{3}(1-\alpha), & 0.3<\alpha
\end{aligned}
$$

with the initial condition $\alpha(x, 0)=0$ and the following definitions:

$$
\begin{aligned}
& \mathrm{K}_{1}=\Delta \mathrm{A}_{1} \cdot \operatorname{Exp}\left(-\Delta \mathrm{E}_{1} / \mathrm{RT}\right) \\
& \mathrm{K}_{2}=\Delta \mathrm{A}_{2} \cdot \operatorname{Exp}\left(-\Delta \mathrm{E}_{2} / R T\right)
\end{aligned}
$$




$$
\mathrm{K}_{3}=\Delta \mathrm{A}_{3} \cdot \operatorname{Exp}\left(-\Delta \mathrm{E}_{3} / \mathrm{RT}\right)
$$

where $\Delta A_{1}, \Delta A_{2}, \Delta A_{3}, \Delta E_{1}, \Delta E_{2}, \Delta E_{3}, \quad R$ and $B$ are material constants, and $T$ is $K^{\circ}$ temperature. Note that the rate of cure presents discontinuity at $\alpha=0.3$.

The second example is taken from the results of compression molding of a polyester [3]. The degree of cure of resin in terms of temperature is given as

$$
\dot{\alpha}=\left(\mathrm{K}_{1}+\mathrm{K}_{2} \alpha^{\mathrm{m}}\right)(1-\alpha)^{\mathrm{n}}
$$

where $m$ and $n$ are constants, and $K_{1}$ and $K_{2}$ are exponential functions of temperature.

Note that in equation (2), the temperature $T_{c}(t)$ on the surface of the piled pre-pregs is called the cure temperature. The cure temperature can be controlled by the processor and is considered as a design variable. Moreover, the performance index of interest is the temperature uniformity $\psi$ which may be defined as the least square of the deviation between the pointwise temperature and the averaged temperature as

$$
\psi=\int_{0}^{T}\left\{\int_{0}^{h} T^{2} d x-\left(\int_{0}^{h} T d x\right)^{2} / h\right\} d t
$$

Some observations of interest are mentioned here:

1. The state equations of the cure process are coupled with two state varlables, the temperature distribution $T(x, t)$ and the degree of cure $\alpha(x, t)$.

2. The nonhomogenous boundary value, $T_{c}(t)$, is the design variable.

3. The rate of cure, $\dot{\alpha}$ in equation (4), exhibits discontinuity, as does the last term $\rho_{\mathrm{R}} \dot{\alpha}$ in the equation of heat conduction.

The heat conduction problem stated in equations (1) to (3) can be simplified to an equation of $\bar{T}(x, t)$,

$$
\rho c \frac{\partial \overline{\mathrm{T}}}{\partial \mathrm{t}}=\mathrm{k} \frac{\partial^{2} \overline{\mathrm{T}}}{\partial \mathrm{x}^{2}}-\rho c \dot{\mathrm{T}}_{\mathrm{c}}+\rho \mathrm{H}_{\mathrm{R}} \dot{\alpha}\left(\overline{\mathrm{T}}+\mathrm{T}_{\mathrm{c}}\right)
$$

with the homogeneous boundary conditions,

$$
\begin{array}{ll}
\frac{\partial \bar{T}(0, t)}{\partial x}=0, & 0<t<T \\
\bar{T}(h, t)=0, & 0<t \leqslant T
\end{array}
$$

and the initial condition,

$$
\overline{\mathrm{T}}(\mathrm{x}, 0)=\mathrm{T}_{\mathrm{o}}(\mathrm{x})-\mathrm{T}_{\mathrm{c}}(0), \quad 0<\mathrm{x}<\mathrm{h},
$$

by introducing the following replacement of the temperature $T(x, t)$ : 


$$
T(x, t)=\bar{T}(x, t)+T_{c}(t)
$$

It is noted that the initial temperature $T_{0}(x)$ of the composite laminate is identical with the initial cure temperature for most applications. Therefore, equation (7) might have not only homogeneous boundary conditions but also a homogeneous initial condition. Moreover, the design variable $T_{c}(t)$ now appears on the right side of equation (7). In other words, the design variable is now involved in terms of heat generation, instead of being a boundary condition. It is also noted that the replacement of $T(x, t)$ doesn't change the structure of the performance index $\psi$, 1.e.,

$$
\psi=\int_{0}^{T}\left\{\int_{0}^{h} \bar{T}^{2} d x-\left(\int_{0}^{h} \bar{T} d x\right)^{2} / h\right\} d t
$$

In general, the cure temperatures recommended by resin manufacturers consist of linear segments. As an example, the cure temperature recommended for the Hercules 3501-6 resin is shown in Fig. 1. Consequently, the right side of heat conduction equation (7) has discontinuous terms of $\rho \mathrm{c} \dot{\mathrm{T}}_{\mathrm{c}}$ as well as $\rho \mathrm{H}_{\mathrm{R}} \dot{\alpha}$. The term $\rho \mathrm{H}_{\mathrm{R}} \dot{\alpha}$ shows discontinuities in both $x$ and $t$ dimensions. The term $\rho c \dot{T}_{c}$, on the other hand, is discontinuous along the $t$ dimension only. Such discontinulties pose numerical difficulties for calculating the design derivatives, especially, when the time or the place at which these discontinuities occur is subjected to change due to the perturbation of the design variable. It is easy to see that the term $\rho H_{R} \dot{\alpha}$ is of this nature. Note that the discontinuity of $\alpha$ is determined by a state variable-dependent jump condition at $\alpha(x, t)=0.3$. Thus, the discontinulty of the term $\mathrm{\rho H}_{\mathrm{R}^{\alpha}} \underset{\sim}{ }$ will take place at the new critical time $\widetilde{t}$ and the new position $\tilde{x}_{\text {so }}$ that $\tilde{\alpha}(\tilde{x}, \tilde{t})=0.3$ for a perturbed state variable $\tilde{\alpha}$. Also the discontinuous point of the term $\rho c T_{c}$ can be shifted, if the time interval of the junction point of constant and variable temperatures of the cure temperature profile, such as $T_{1}$ in Fig. 1, is considered as a design variable.

\section{LOGICAL FUNCTION MODELLING}

Quite a few engineering examples whose state variables show discontinuities in derivatives can be found in the multi-state control problems [8], and the mechanical systems with intermittent motion $[9,10]$. However, the derivative discontinuities of those examples are associated with time dimension only.

The intermittent motion is characterized by the occurrence of nearly discontinuous force and velocity caused by impulsive force, impact, mass capture, and mass release. The optimal design problems of mechanisms with intermittent motion have been discussed by Huang, Huag and Andrews [9]. The1r method is based on the identification of critical times at which discontinuities in forces or velocities occur [8]. The overall time interval of analysis can be divided into a number of subintervals based on those critical times. The jump conditions of state variables are then employed in an adjoint variable approach to determine the discontinuities of adjoint variables. The adjoint variables are then used for the calculation of design sensitivity coefficients. In employing this approach, an a priori knowledge of the critical times is required. The determination of the critical times of jump conditions, however, may lead to a rather complex logic for 
digital computer programming. In order to avoid these complexities, Ehle and Huag [10] introduced a "logical function" to smoothly approximate discontinuities, and then calculated the design derivatives by the standard adjoint variable technique. An example in their work shows that the proper selection of the sizes of the time step and the transient zone used for discontinuity approximation is crucial to the accuracy of design sensitivity calculation. However, making such a selection is difficult. Nevertheless, the logical function approach is used in this study. The reason is that $\dot{\alpha}$ is a function of time as well as spatial position. As a result, keeping track of the $\dot{\alpha}$ discontinuity at every spatial position is a very difficult task for numerical analysis.

As mentioned earlier, logical functions can be used to represent a sequence of logical events. A $\operatorname{logical}$ function $L(z, \varepsilon)$ is a continuous function which smoothly approximates a Heaviside step function $H(z)$ within a given region $0 \leqslant z<\varepsilon$ for a small number $\varepsilon$. The symmetrical step function $H(z)$ is defined as:

$$
H(z)=\begin{array}{ll}
0, & z<0 \\
\frac{1}{2}, & z=0 \\
1, & z>0 .
\end{array}
$$

The logical function employed here is given in reference [10] as:

$$
L(z, \varepsilon)=\frac{1}{2} \frac{|z|^{2 n+1}+z^{2 n+1}}{|z|^{2 n+1}+\frac{1}{2}\left[|z-\varepsilon|^{2 n+1}-(z-\varepsilon)^{2 n+1}\right]}
$$

where $\mathrm{n}$ is an integer selected in order to ensure the continuity of the derivative up to order $d$, i.e., $2 n+1>d$. The $n$ is taken as 1 in this study. The approximation of the logical function is shown in Fig. 2. Note that the values of a logical function $L(z, \varepsilon)$ are $0,1 / 2$ and 1 for $z=0, \varepsilon / 2$ and $\varepsilon$, respectively, and the transition width $\varepsilon$ defines the region of approximation. The value of the logical function is exactly identical with the Heaviside step function outside the approximation region.

In using the logical function method, one is free to choose a wide variety of arguments that determine the transition point for a logical function. As an example, the transition condition $\alpha=0.3$ for the degree of cure can be used to define a logical function $\mathrm{L}(\alpha-0.3, \varepsilon)$ such that $\mathrm{L}(\alpha-0.3, \varepsilon)=1$ when $\alpha>0.3+\varepsilon$, and $\mathrm{L}(\alpha-0.3, \varepsilon)=0$ when $\alpha \leqslant 0.3$. Based on this definition, the logical function can be used to compress the equation of cure kinetics into a compact form:

$$
\dot{\alpha}=\mathrm{f}_{1} \cdot[1-\mathrm{L}(\alpha-0.3, \varepsilon)]+\mathrm{f}_{2} \cdot \mathrm{L}(\alpha-0.3, \varepsilon)
$$

Note that the above single equation of cure kinetics is the same as the original equation over the entire time interval of analysis outside the transition period. Furthermore, since the logical function is a smooth function of $\alpha$, there is no discontinuity in the $\alpha$ of the preceding equation. Thus, the analysis of the design sensitivity can be simplified to a great extent, because there is no need to monitor the perturbation of $\alpha$ discontinuity. Note that the value of $\alpha(x, t)$ in equation (12) can be calculated by the linear combination of shape functions and nodal values obtained by the finite element analysis. Similarly, the discontinuity in $\dot{\mathrm{T}}_{\mathrm{c}}$ can be smoothed out in the same manner. Again, using the cure temperature profile 
indicated in figure 1 as an example, the first discontinuity at $t=\mathrm{T}_{1}$ can be expressed as

$$
\dot{\mathrm{T}}_{\mathrm{c}}=\mathrm{a}\left[1-\mathrm{L}\left(\mathrm{t}-\mathrm{T}_{1}, \varepsilon\right)\right]
$$

It is then easy to consider the junction point $T_{1}$ as a design variable based upon the above equation.

Finally, the heat conduction equation stated in equation (7) can be expressed as

$$
\begin{aligned}
& \rho c \frac{\partial \bar{T}}{\partial t}=k \frac{\partial^{2} \bar{T}}{\partial x^{2}}+g\left(\bar{T}, \alpha, T_{1}\right) \\
& =k \frac{\partial^{2} \bar{T}}{\partial x^{2}}-\rho c a \cdot\left[1-L\left(t-T_{1}, \varepsilon\right)\right] \\
& +\mathrm{pH}_{\mathrm{R}} \mathrm{f}_{1} \cdot[1-\mathrm{L}(\alpha-0.3, \varepsilon)]+\mathrm{pH}_{\mathrm{R}} \mathrm{f}_{2} \cdot \mathrm{L}(\alpha-0.3, \varepsilon)
\end{aligned}
$$

for the cure cycle given in figure 1. Similarly, the equation of the degree of cure given in equation (12) can be rewritten here,

$$
\begin{aligned}
\dot{\alpha} & =\mathrm{f}\left(\overline{\mathrm{T}}, \alpha, \mathrm{T}_{1}\right) \\
& =\mathrm{f}_{1} \cdot[1-\mathrm{L}(\alpha-0.3, \varepsilon)]+\mathrm{f}_{2} \cdot \mathrm{L}(\alpha-0.3, \varepsilon)
\end{aligned}
$$

The finite element discretization is then introduced to convert the above initial-boundary value equations into a set of first order differential equations:

and

$$
[C]\{\dot{\bar{T}}\}+[K]\{\overline{\mathrm{T}}\}=\{\mathrm{F}(\{\overline{\mathrm{T}}\},\{\alpha\})\}
$$

$$
[N]\{\dot{\alpha}\}=\{G(\{\overline{\mathrm{T}}\},\{\alpha\})\}
$$

Quadratic and Iinear polynomials are used to interpolate the states of temperature distribution and degree of cure, respectively. Note that the right side vectors of the above two matrix equations are different. This is because the trial functions for equations (14) and (15) are different.

The finite-element discretization can also be used to simplify the expression for the performance index of concern into a single integral:

$$
\psi=\int_{0}^{\mathrm{T}}\left(\{\overline{\mathrm{T}}\}^{\mathrm{T}}[\mathrm{C}]\{\overline{\mathrm{T}}\}-\{\overline{\mathrm{T}}\}^{\mathrm{T}}\{\mathrm{P}\}\{\mathrm{P}\}^{\mathrm{T}}\{\overline{\mathrm{T}}\} / \mathrm{h}\right) d t
$$

where the [C] is same as the one defined in equation (16), and the components of the vector $\{\mathrm{P}\}$ are obtained by integrating the quadratic shape functions of temperature.

This set of equations (16) and (17) is then solved simultaneously by a numerical integration code called DE [11]. The DE program is one of predictor- 
corrector integration algorithms using the Adams family of formulas. The truncation error is controlled by varying the step size and the order of the method. The DE program has the capability to handle moderately stiff equations which of ten occur in the problems of chemical kinetics. To preserve the accuracy of analysis, the temperature distribution and the degree of cure are subjected to the same numerical error tolerance during the numerical integration.

\section{DESIGN SENSITIVITY ANALYSIS}

In general, there are four ways to calculate the thermal design derivatives, i.e., the finite-difference method, Green's function approach, the direct differentiation method and the adjoint variable technique. The last two are of ten mentioned in the literature [12-15]. Both methods lead to a set of linear equations that have a structure similar to the original system.

The computational efforts regarding the direct differentiation method and the adjoint variable method depend mainly on the numbers of constraints and design variables of concern. The direct differentiation method requires the solution of a differential equation for each design variable; while the adjoint variable method requires the solution of an adjoint equation for each constraint. Consequently, the direct differentiation method is more efficient in calculating the design derivatives than the adjoint variable method when the number of design variables is less than the number of constraints, or vice versa.

It is known that the direct differentiation method provides equations of design derivatives which can be integrated forward, instead of backward to solve the adjoint variables. The equations of design derivatives can, therefore, be solved simultaneously with the original system of equations and are subjected to the same numerical error tolerance. Furthermore, the approach of direct differentiation provides, without extra efforts, the time histories of design derivatives of functionals and state variables. This information can be used by a designer to reconstruct the design space. One may check this information to see whether a design variable of concern contributes to the perturbation of the performance index consistently over a long or short period of time. As an example, the time histories of design derivatives of various pollutants' concentrations with respect to emission and meteorological parameters are studied and used in reference [16] to improve the mathematical model of air quality. In this study, the direct differentiation method and the adjoint variable technique, in conjunction with the logical function method, is used for the calculation of thermal design sensitivities.

The calculation of design derivatives using the direct differentiation method is straightforward. For example, let $\mathrm{T}_{1}$ in Fig. 1 be the design variable. The direct differentiation of equations (14) and (15) yields

$$
\begin{aligned}
\rho c \frac{\partial \overline{\mathrm{T}}^{\prime}}{\partial t} & =k \frac{\partial^{2} \overline{\mathrm{T}}^{\prime}}{\partial \mathrm{x}^{2}}-\rho c \text { a } \mathrm{L}^{\prime}\left(\mathrm{t}-\mathrm{T}_{1}, \varepsilon\right) \\
& +\rho \mathrm{H}_{\mathrm{R}}\left(\frac{\partial \mathrm{f}}{\partial \alpha} \alpha^{\prime}+\frac{\partial \mathrm{f}_{1}}{\partial \overline{\mathrm{T}}} \mathrm{T}^{\prime}\right) \cdot[1-\mathrm{L}(\alpha-0.3, \varepsilon)]
\end{aligned}
$$




$$
+\rho \mathrm{R}_{\mathrm{R}}\left(\frac{\partial \mathrm{f}_{2}}{\partial \alpha} \alpha^{\prime}+\frac{\partial \mathrm{f}_{2}}{\partial \overline{\mathrm{T}}} \overline{\mathrm{T}}^{\prime}\right) \mathrm{L}(\alpha-0.3, \varepsilon)+\rho \mathrm{R} \frac{\mathrm{dL}}{\mathrm{d} \alpha} \alpha^{\prime} \cdot\left(\mathrm{f}_{2}-\mathrm{f}_{1}\right)
$$

and

$$
\begin{aligned}
\dot{\alpha}^{\prime} & =\left(\frac{\partial f_{1}}{\partial \alpha} \alpha^{\prime}+\frac{\partial f_{1}}{\partial \bar{T}} T^{\prime}\right) \cdot[1-L(\alpha-0.3, \varepsilon)] \\
& +\left(\frac{\partial f_{2}}{\partial \alpha} \alpha^{\prime}+\frac{\partial f_{2}}{\partial \bar{T}} \bar{T}^{\prime}\right) \cdot L(\alpha-0.3, \varepsilon) \\
& +\frac{d L}{d \alpha} \alpha^{\prime}\left(f_{2}-f_{1}\right)
\end{aligned}
$$

where the prime indicates the design derivative with respect to $T_{1}$. The derivative of the logical function $\mathrm{dL} / \mathrm{d} \alpha$ is an approximation of a delta function which can be derived from the definition of the logical function L. From equations (8-9) and the initial condition of $\alpha(x, 0)=0$, the boundary and initial conditions for design derivatives, $\overline{\mathrm{T}}^{\prime}$ and $\alpha^{\prime}$, can be derived as

$$
\begin{array}{ll}
\frac{\partial \bar{T}^{\prime}(o, t)}{\partial x}=0, & 0<t<T \\
\bar{T}^{\prime}(h, t)=0, & 0<t<T
\end{array}
$$

and

$$
\begin{array}{ll}
\bar{T}^{\prime}(x, 0)=-T_{c}^{\prime}(0), & 0<x<h \\
\alpha^{\prime}(x, 0)=0 & ,
\end{array}
$$

where $\mathrm{T}_{c}^{\prime}(0)$ is usually zero unless the initial control temperature $\mathrm{T}_{c}{ }^{\prime}(0)$ is considered as a design variable. With these boundary and inftial conditions, the last two coupled linear equations can be solved numerically for the design derivatives $\overline{\mathrm{T}}^{\prime}$ and $\alpha^{\prime}$.

Based on the same finite element discretization as used in solving the original system, equations (19) and (20) can be converted into a set of linear ordinary differential equations:

and

$$
[C]\left\{\overline{\mathrm{T}}^{\prime}\right\}+[\mathrm{K}]\left\{\overline{\mathrm{T}}^{\prime}\right\}=\left\{\mathrm{H}\left(\{\overline{\mathrm{T}}\},\{\alpha\},\left\{\overline{\mathrm{T}}^{\prime}\right\},\left\{\alpha^{\prime}\right\}\right)\right\}
$$

$$
[\mathrm{N}]\left\{\dot{\alpha}^{\prime}\right\}=\left\{\mathrm{Q}\left(\{\overline{\mathrm{T}}\},\{\alpha\},\left\{\overline{\mathrm{T}}^{\prime}\right\},\left\{\alpha^{\prime}\right\}\right)\right\}
$$

Note that the coefficient matrices of equations (23) and (24) are simflar to those of equations (16) and (17). However, $\{\overline{\mathrm{T}}\}$ and $\{\alpha\}$ appear in equations (23) and 
(24). Thus, the numerical integration of equations (16) - (17) and (23) - (24) can be performed simultaneously so as to maintain equal accuracy between state variables $(\{\overline{\mathrm{T}}\},\{\alpha\})$ and design derivatives $\left(\left\{\overline{\mathrm{T}}^{\prime}\right\},\left\{\alpha^{\prime}\right\}\right)$. The DE program, mentioned previously, is employed as an integrator to obtain the numerical results of design derivatives.

The values of the design derivative of temperature $\left\{\bar{T}^{\prime}\right\}$ can then be directly substituted into the following equation to calculate the thermal design derivative of the performance index:

$$
\left.\psi^{\prime}=2 \int_{0}^{T}\{\bar{T}\}^{T}[C]\left\{\bar{T}^{\prime}\right\}-\{\bar{T}\}^{T}\{P\}\{P\}^{T}\left\{\bar{T}^{\prime}\right\} / h\right) d t
$$

The above equation is derived from equation (18) by using the direct differentiation me thod.

Regarding the computational efficiency of the direct differentiation method, it is worthwhile mentioning two notes here:

1. Because the coefficient matrices of $\left\{\dot{\bar{T}}^{\prime}\right\}$ and $\left\{\dot{\alpha}^{\prime}\right\}$ are identical to those of $\{\dot{\bar{T}}\}$ and $\{\dot{\alpha}\}$, the triangular factorizations of matrices [C] and [M] need to be done once only. The calculation of $\{\dot{\bar{T}}\}$ and $\{\dot{\alpha}\}$ can be carried out by back substitution for each of design variables.

2. Compared to the original system equations, the right side of equations for computing $\left\{\overline{\mathrm{T}}^{\prime}\right\}$ and $\left\{\alpha^{\prime}\right\}$, such as equations (23) and (24), may have different frequency contents. Thus, to maintain the same numerical accuracy, a smaller time step $\Delta t$ may be required for the $D E$ program to solve the pairs $(\{\bar{T}\},\{\alpha\})$ and $\left(\left\{\bar{T}^{\prime}\right\},\left\{\alpha^{\prime}\right\}\right)$ simultaneously.

A major step in the adjoint variable method is deriving the adjoint equations to solve the design derivatives of equations (18) in terms of state and adjoint variables. In order to do so, one may extend the performance index $\psi$ of equation (11) as, using $\mathrm{T}_{1}$ as a design variable,

$$
\begin{aligned}
& \psi=\int_{0}^{T} \int_{0}^{h}\left\{\left[\bar{T}-\int_{0}^{h} \frac{\bar{T}}{h} d x\right] \bar{T}\right\} d x d t \\
& \left.=\int_{0}^{T} \int_{0}^{h}\left\{\bar{T}-\int_{0}^{h} \bar{T} \frac{\bar{T}}{h} d x\right] \bar{T}\right\} d x d t \\
& +\int_{0}^{T} \int_{0}^{h}\left\{\lambda\left[\rho c \frac{\partial \bar{T}}{\partial t}-k \frac{\partial^{2} \bar{T}}{\partial x^{2}}-g\left(\bar{T}, \sigma, T_{1}\right)\right]+\right. \\
& \left.s\left[\frac{\partial \alpha}{\partial t}-f\left(\bar{T}, \alpha, T_{1}\right)\right]\right\} d x d t
\end{aligned}
$$


where $\alpha(x, t)$ and $s(x, t)$ are two arbitrary functions. Note that the last integral is zero because of state equations (14-15). Taking the design derivative of the above equation with respect to $\mathrm{T}_{1}$ and integrating by parts, it follows that

$$
\begin{aligned}
\psi^{\prime} & =\int_{0}^{T} \int_{0}^{h}\left(-\lambda \frac{\partial g}{\partial T_{l}}-s \frac{\partial f}{\partial T_{1}}\right) d x d t \\
& \left.+\int_{0}^{T} \int_{0}^{h}\left[-\rho c \frac{\partial \lambda}{\partial t}-k \frac{\partial^{2} \lambda}{\partial x^{2}}-\lambda \frac{\partial g}{\partial \bar{T}}-s \frac{\partial f}{\partial \bar{T}^{\prime}}+2\left(\overline{T^{\prime}}-\int_{0}^{h} \frac{\bar{T}}{h} d x\right)\right] \overline{T^{\prime}}\right\} d x d t \\
& +\int_{0}^{T} \int_{0}^{h}\left\{\left[-\frac{\partial s}{\partial t}-s \frac{\partial f}{\partial \alpha}-\lambda \frac{\partial g}{\partial \alpha}\right] \alpha^{\prime}\right\} d x d t \\
& +\left.\int_{0}^{T}\left(-k \lambda \frac{\partial \bar{T}^{\prime}}{\partial x}+k \frac{\partial \lambda}{\partial x} \bar{T}^{\prime}\right)\right|_{0} ^{h} d t \\
& +\left.\int_{0}^{h}\left(\rho c \lambda \bar{T}^{\prime}+s \alpha^{\prime}\right)\right|_{0} ^{T} d x
\end{aligned}
$$

Note that the only two unknowns in the above equation are the design derivatives $\bar{T}^{\prime}$ and $\alpha^{\prime}$. One may now specify the variables $\lambda$ and $s$ in such a way that all terms assoclated with $T^{\prime}$ and $\alpha^{\prime}$ are dropped. This can be accomplished by introducing the following adjoint equations for $\lambda$ and $s$ :

and,

$$
0=\rho c \frac{\partial \lambda}{\partial t}+k \frac{\partial^{2} \lambda}{\partial x^{2}}+\lambda \frac{\partial g}{\partial \bar{T}}+s \frac{\partial f}{\partial \bar{T}}-2\left(\bar{T}-\int_{0}^{h} \frac{\bar{T}}{h} d x\right)
$$

$$
0=\frac{\partial s}{\partial t}+\lambda \frac{\partial g}{\partial \alpha}+s \frac{\partial f}{\partial \alpha}
$$

with the terminal conditions,

$$
\begin{array}{ll}
\lambda(x, T)=0, & 0<x<h, \\
s(x, T)=0 & 0<x<h,
\end{array}
$$

and the boundary conditions,

$$
\begin{aligned}
\frac{\partial \lambda}{\partial x}(0, t) & =0 & & 0<t<T, \\
\lambda(h, t) & =0, & & 0<t<T,
\end{aligned}
$$

Then, the combination of equations (25 - 31) provides a simple formula for the design derivative of the functional, 


$$
\begin{aligned}
\psi^{\prime} & =\int_{0}^{T} \int_{0}^{h}\left(-\lambda \frac{\partial g}{\partial T_{1}}-s \frac{\partial f}{\partial T_{1}}\right) d x d t \\
& +\int_{0}^{h} \rho c \lambda(x, 0) T_{c}^{\prime}(0) d x
\end{aligned}
$$

Equation (32) shows that the design derivative of $\psi$, namely, $\psi^{\prime}$, is a functional of the state variables $\alpha$ and $\vec{T}$, and the adjoint variables $\lambda$ and $s$. Since the adjoint variables of equations $(26-27)$ form an "adjoint" diffusionreaction system similar to the original one, the same numerical scheme used to solve the state variables $\alpha$ and $\overline{\mathrm{T}}$ can be extended here to compute - the adjoint variables $s$ and $\lambda$. For instance, using the shape functions of $\alpha$ and $\bar{T}$ in equations $(16$ - 17) to interpolate the adjoint variables $\lambda$ and $s$ obtains the following matrix equations for nodal values of $\lambda$ and $s$,

$$
\begin{aligned}
& {[\mathrm{C}]\{\dot{\lambda}\}=[\mathrm{K}]\{\lambda\}+\{\mathrm{R}(\{\overline{\mathrm{T}}\},\{\alpha\},\{\lambda\},\{\mathrm{s}\})\}} \\
& {[\mathrm{N}]\{\dot{\mathrm{s}}\}=\{\mathrm{S}(\{\overline{\mathrm{T}}\},\{\alpha\},\{\lambda\},\{\mathrm{s}\})\}}
\end{aligned}
$$

with the proper boundary and terminal conditions.

In general, the adjoint equations cannot be solved simultaneously with the original system equations. Because of the terminal conditions, the adjoint equations can be solved by either the backward integration along the real-time taxis directly or the forward integration along the artificial time $t^{*}$-axis by changing the independent variable $t$ to $t^{*}$ as $t^{\star}=T-t$. However, both approaches require the solutions of the original system equations prior to solving the adjoint equations.

In the derivation of design derivatives, it has been assumed that $T(x, b, t)$ and $\alpha(\mathrm{x}, \mathrm{b}, \mathrm{t})$ have enough regularity in the time-spatial domain and in the design space.

\section{NUMERICAL EXAMPLES AND RESULTS}

Four examples are presented in this section to discuss the numerical accuracy of the logical function approximation and the methods for calculating the thermal design derivatives. The accuracy of the thermal design sensitivity analysis is checked, based on the fundamental definition of design derivatives which states that they can be approximated by the finite difference. In other words, it is mathematically true for a small perturbation of design variable $\Delta \mathrm{T}_{\mathrm{c}}$ so that:

$$
\psi^{\prime} \equiv \frac{\mathrm{d} \psi}{\mathrm{dT}} \cong \frac{\Delta \psi}{\Delta \mathrm{T}_{\mathrm{c}}}
$$

The perturbation of the design variable $\Delta \mathrm{T}_{c}$ is defined as the difference between a perturbed design $\mathrm{T}_{c}{ }^{*}$ and the nominal design $\mathrm{T}_{c}$, i.e.,

$$
\Delta \mathrm{T}_{\mathrm{c}}=\mathrm{T}_{\mathrm{c}}^{*}-\mathrm{T}_{\mathrm{c}}
$$


As a result of the above definitions, it follows that

$$
\begin{aligned}
\Delta \psi & \equiv \psi\left(\mathrm{T}_{\mathrm{c}}{ }^{*}\right)-\psi\left(\mathrm{T}_{\mathrm{c}}\right) \\
& \simeq \psi^{\prime} \cdot \Delta \mathrm{T}_{\mathrm{c}}
\end{aligned}
$$

The above equation provides a simple means to check the accuracy of the design sensitivity analysis.

The first example presented here deals with the curing process of compression molding (equation (1) and (5)) in which the cure temperature of the process is assumed to be a constant temperature. The nominal cure temperature is taken as $423^{\circ} \mathrm{K}$, and there is no discontinulty involved. According to the approximation defined in equation (35), the results shown in figure 3 demonstrate the validity of the direct differentiation method for the thermal design sensitivity analysis.

The second example, on the other hand, refers to the curing process of press processing (equation (1) and (4)) in which a jump condition appears in the derivative of the degree of cure. The profile of the cure temperature is assumed to be $T_{c}(t)=b_{0}+b_{1} t$ where the initial temperature $b_{0}$ and heating rate $b_{1}$ are considered as design variables. The nominal values of $b_{0}$ and $b_{1}$ are taken as $290^{\circ} \mathrm{K}$ and $1.7^{0} \mathrm{~K} / \mathrm{sec}$. The changes of the performance index with respect to the values of $b_{0}$ and $b_{1}$ are calculated by using the direct differentiation method as

$$
\begin{aligned}
& \Delta \psi=-0.3158 \cdot \Delta \mathrm{b}_{0} \\
& \Delta \psi=2.6336 \cdot \Delta \mathrm{b}_{1}
\end{aligned}
$$

However, using the adjoint variable technique obtains

$$
\begin{aligned}
& \Delta \psi=-0.0367 . \Delta \mathrm{b}_{0} \\
& \Delta \psi=8.099 \cdot \Delta \mathrm{b}_{1}
\end{aligned}
$$

The results indicated in Tables 1 and 2 show that the direct differentiation method, in conjunction with the logical function approximation, performs very well even for a thermal problem with discontinuous derivatives. It is also shown in Table 2 that the relation between the performance index and the heating rate $b_{1}$ is highly nonlinear. In this example, the transient width $\varepsilon$ of equation (15) is defined as $10^{-4}$ second which is the smallest time step size allowed in the DE program.

Next, the thermal design derivative of the compression molding is studied, with the cure temperature being given in figure 4. The value of $T_{1}$, where the rate of the cure temperature changes, is considered as a design variable. In this study, the nominal value and the perturbation of $\mathrm{T}_{1}$ are taken as 40 seconds and 1 second, respectively. The discontinuity of $\dot{\mathrm{T}}_{c}$ at $\mathrm{T}_{1}$ can be smoothed by equation (13). The upper curve shown in figure 5 is obtained by using the direct differentiation method based on equation (13). On the other hand, the lower curve displayed in figure 5 is obtained by using the following expression for the design derivative of $\dot{T}_{c}$ : 


$$
\frac{\mathrm{dT}_{\mathrm{c}}}{\mathrm{dT}_{1}}= \begin{cases}0, & t \leqslant \mathrm{~T}_{1} \\ \mathrm{a}, & t>\mathrm{T}_{1}\end{cases}
$$

The design derivative of $\dot{T}_{c}$ at the junction point, $T_{1}$, is a delta function which is not included in the above equation. The results in figure 5 clearly show that the design derivative of the jump condition should be considered in the sensitivity calculation.

It is easy to obtain the time histories of design derivatives of state variables using the direct differentiation approach. Using this information, the processor can investigate whether a design variable of concern contributes to the change of system performance consistently over a long or short period of time so as to reconstruct the design space. For example, figures 6 and 7 show that the change of the design variable $\mathrm{T}_{1}$ has a significant effect on the temperature and the degree of cure on the surface of the pre-pregs when the time is 42 seconds.

The distribution of thermal design derivatives $\alpha^{\prime}$ and $T^{\prime}$ along the thickness of the pre-pregs is shown in figures 8 and 9 for different instants of time. It is of great interest to see that the most significant changes of $T$ and $\alpha$ due to the change of the junction point $T_{1}$ happen around 80 seconds and at $2.5 \mathrm{~mm}$ from the surface of pre-pregs.

In this example, the various values of transient width, regioned from $10^{-2}$ second to $10^{-4}$ second are chosen to be used in the logical function approximation. The sensitivity results obtained accordingly are essentially the same. This indicates that the value of the transition width in the range of study has no significant effect on the accuracy of the sensitivity analysis. The transition width $\varepsilon$ is taken as $10^{-3}$ second in the results reported in figures 6 to 9 .

Finally, the cure temperature of the press process studied herein is again the same as the one shown in figure 1 . With 100 minutes as the nominal value, $\mathrm{T}_{1}$ is considered as a design variable. Thus, both equations (12) and (13) should be used to approximate the jumps in $\dot{\alpha}$ and $\dot{T}_{c}$ smoothly for the thermal problem of the press process. The results of sensitivities calculated by the direct differentiation method are in good agreement with the actual changes calculated by the finite difference method as shown in figure 10. The transition regions used in this example are $10^{-4}$ second and $10^{-2}$ second for equations (12) and (13), respectively.

\section{vi. CONCLUSIONS}

It is quite common to have empirical formulations appear in the state equations modelling the composite curing process. These empirical formulations may introduce discontinuous state derivatives into the state equations. A simple method which uses the logical function approximation is introduced in this paper to perform the thermal design sensitivity analysis for such state equations.

Based on the numerical study, it is obvious that the direct differentiation method provides more accurate results than the adjoint variable method does. The direct differentiation method also yields the time histories of the design derivatives. In addition, the information of design derivatives of the pointwise 
constraints can be obtained by using the direct differentiation method without extra cost. It is thus concluded that for the transient problem in this study, the direct differentiation method is superior to the adjoint variable technique in terms of accuracy and physical interpretation of results.

\section{REFERENCES}

1. Loos, A. C., and Springer, G. S., "Curing of Epoxy Matrix Composites," J. of Composite Materials, Vol. 17, pp. 135-169, 1983.

2. Lee, W. I., Loos, A. C., and Springer, G. S., "Heat of Reaction, Degree of Cure, and Viscosity of Hercules 3501-6," J. of Composite Materials, Vol. 16, pp. $510,1982$.

3. Barone, M. E., and Caulk, D. A., "The Effect of Deformation and Thermoset Cure on Heat Conduction in a Chopped-Fiber Reinforced Polyster During Compression Molding," Int. J. Heat Mass Transfer, Vol. 22, pp. 1021-1032, 1979.

4. Hou, J. W., "Optimal Cure Cycle Design for Autoclave Processing of Thick Composite Laminates: A Feasibility Study," Progress Report, ODU Research Foundation, November 1985.

5. Haftka, R. T., "Techniques for Thermal Sensitivity Analysis," Int. J. Num. Meth. Engng., Vo1. 17, pp. 71-80, 1981 .

6. Biegler, L. T., "Solution of Dynamics Optimization Problems by Successive Quadratic Programming and Orthogonal Allocation," Report No. DRC-06-45-83, Design Research Center, Carnegie-Mellon University, Pittsburgh, PA, 1983.

7. Irwin, C. L., and Komkov, V., "Sensitivity Analysis and Model Optimization for Reaction-Diffusion Systems," Journal of Optimization Theory and Applications, Vo1. 44, No. 4, pp. 569-584, 1984.

8. Bryson, A. E., and Ho, Y. C., Applied Optimal Control, Ginn and Co., MA, 1969.

9. Huang, R. C., Haug, E. J. and Andrews, J. G., "Sensitivity Analysis and Optimal Design of a Mechanical System with Intermittent Motion," ASME J. Mech. Design, Vol. 100, No. 3, pp. 492-499, 1978.

10. Ehle, P. E. and Haug, E. J., "A Logical Function Method for Dynamic and Design Sensitivity Analysis of Mechanical System with Intermittent Motion," ASME J. Mech. Design, Vo1. 104, No. 1, pp. 90-100, 1982.

11. Shampine, L. F., and Gordon, M. K., Computer Solution of Ordinary Differential Equations: The Initial Value Problem, W. H. Freeman and Co., San Francisco, CA., 1975.

12. Adelman, H. M., Haftka, R. T., Camarda, C. J., and Wa1sh, J. L., "Structural Sensitivity Analysis: Methods, Applications and Needs," NASA TM-85827, June 1984 . 
13. Hsieh, C. C., and Arora, J. S, "Design Sensitivity Analysis and Optimization of Dynamic Response," Computer Methods in Applled Mechanics and Engineering, No. 2, Vol. 43, pp. 195-219, 1984.

14. Haug, E. J., Mani, N. K., and Krishnaswami, P., "Design Sensitivity Analysis and Optimization of Dynamically Driven Systems," Computer-Aided Analysis and Optimization of Mechanical System Dynamics, (ed. E. J. Haug) Springer-Verlag, 1984 .

15. Krishnaswami, P., Wahage, R. A., and Haug, E. J., "Design Sensitivity Analysis of Constrained Dynamic Systems of Direct Differentiation," Technical Report No. 83-5, Center for Computer-Aided Design, The University of Iowa, Iowa City, 1983.

16. Tilden, J. W., and Seinfeld, J. H., "Sensitivity Analysis of a Mathematical Model for Photochemical Air Pollution," Atmospheric Environment, Vol. 16, No. 6, pp. 1357-1364, 1982 . 
TABLE I DESIGN SENSITIVITY RESULTS FOR DESIGN

VARIABLE $b_{0}$ IN EXAMPLE 1

\begin{tabular}{lllll}
\hline \hline & & & \multicolumn{2}{c}{$\psi^{1} \cdot \Delta \mathrm{b}_{0}$} \\
$\mathrm{~b}_{0}$ & $\psi$ & $\Delta \psi$ & (Direct Diff) & (Adjoint) \\
\hline 290.0 & 26.217 & -- & -- & 0.0037 \\
289.9 & 26.248 & 0.0315 & 0.0316 & 0.0073 \\
289.8 & 26.280 & 0.0628 & 0.0632 & 0.0110 \\
289.7 & 26.311 & 0.0940 & 0.0948 & 0.0183 \\
289.5 & 26.313 & 0.1559 & 0.1579 & 0.0367 \\
289 & 26.525 & 0.3077 & 0.3157 & \\
\hline
\end{tabular}

TABLE 2 DESIGN SENSITIVITY RESULTS FOR DESIGN

VARIABLE $b_{1}$ IN EXAMPLE 1

\begin{tabular}{lllll}
\hline \hline & & & \multicolumn{2}{c}{$\psi^{1} \cdot \Delta \mathrm{b}_{1}$} \\
$\mathrm{~b}_{1}$ & $\psi$ & $\Delta \psi$ & (Direct Diff.) & (Adjoint) \\
\hline 1.700 & 26.217 & -- & - & - \\
1.702 & 26.222 & 0.00497 & 0.00527 & 0.01620 \\
1.706 & 26.230 & 0.01312 & 0.01580 & 0.04859 \\
1.708 & 26.233 & 0.01633 & 0.02107 & 0.06479 \\
1.710 & 26.236 & 0.01898 & 0.02637 & 0.08099 \\
1.730 & 26.234 & 0.01713 & 0.07901 & 0.24297 \\
1.750 & 26.189 & -0.02805 & 0.13168 & 0.4050 \\
1.800 & 26.962 & -0.25457 & 0.26336 & 0.8099 \\
\hline
\end{tabular}




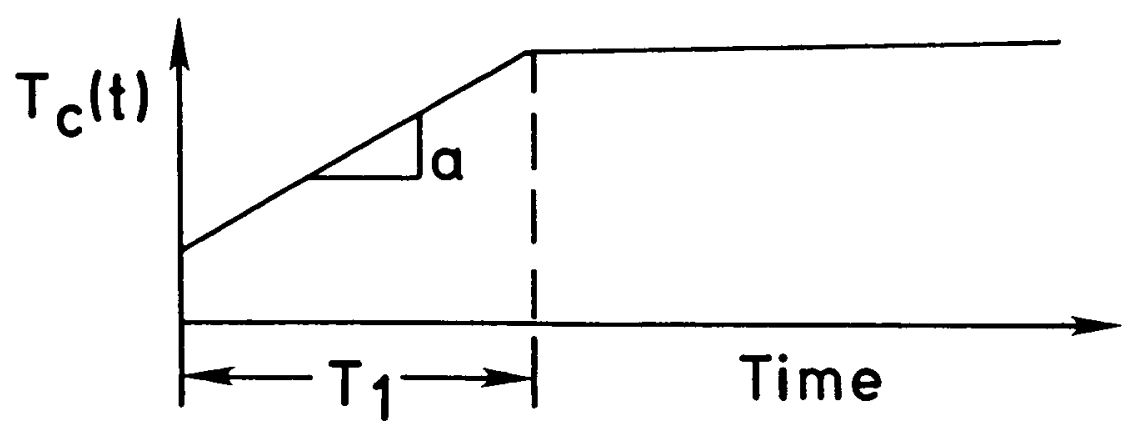

Figure 1. A typical cure temperature.

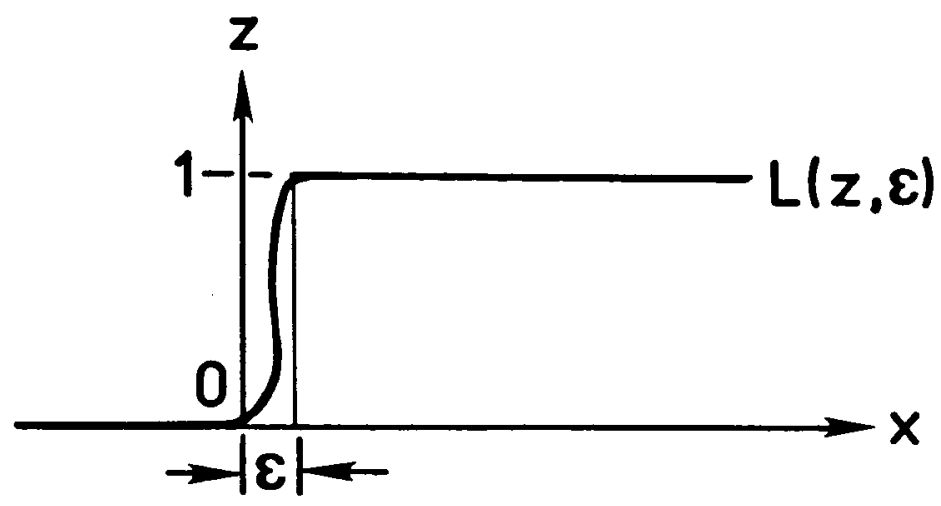

Figure 2. The approximation of logical function.

$$
\text { c }-4
$$




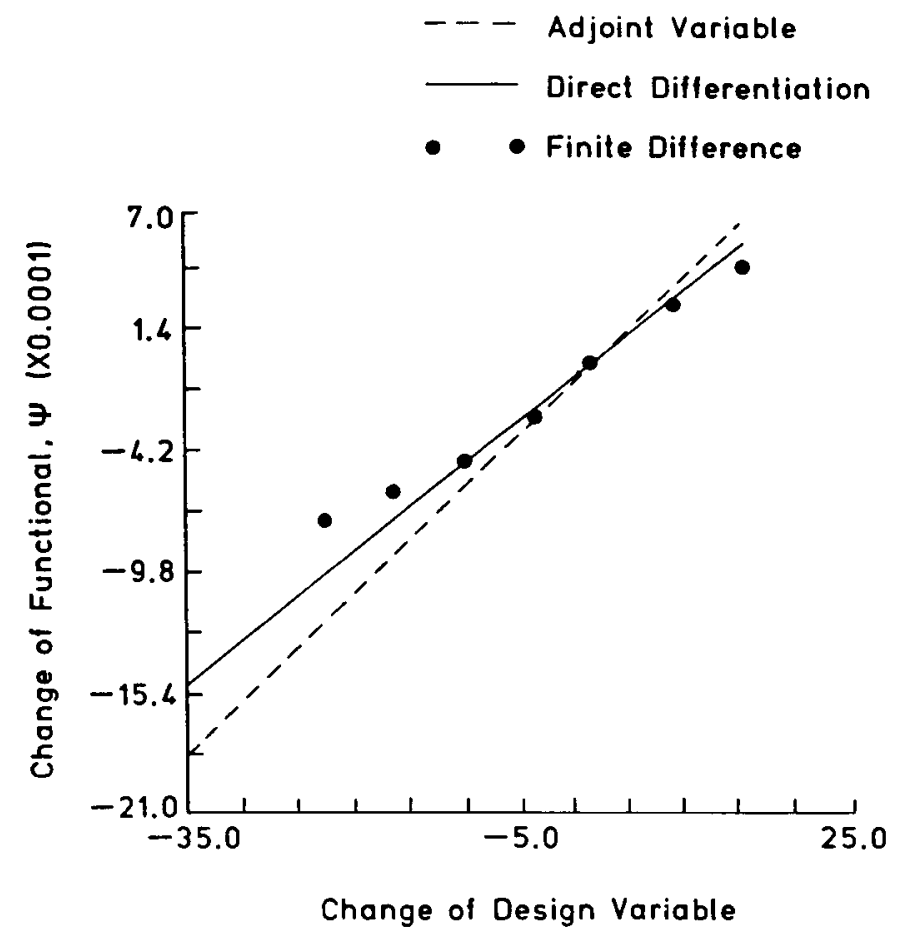

Figure 3. Thermal design derivatives for compression molding with respect to the mold temperature.

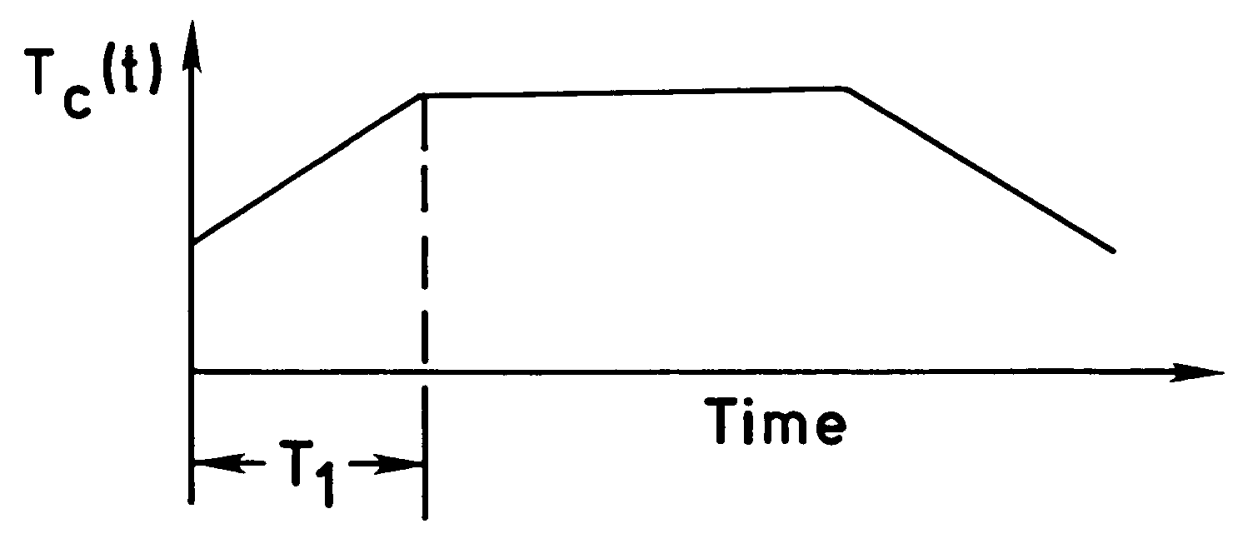

Figure 4. The cure temperature for compression molding. 


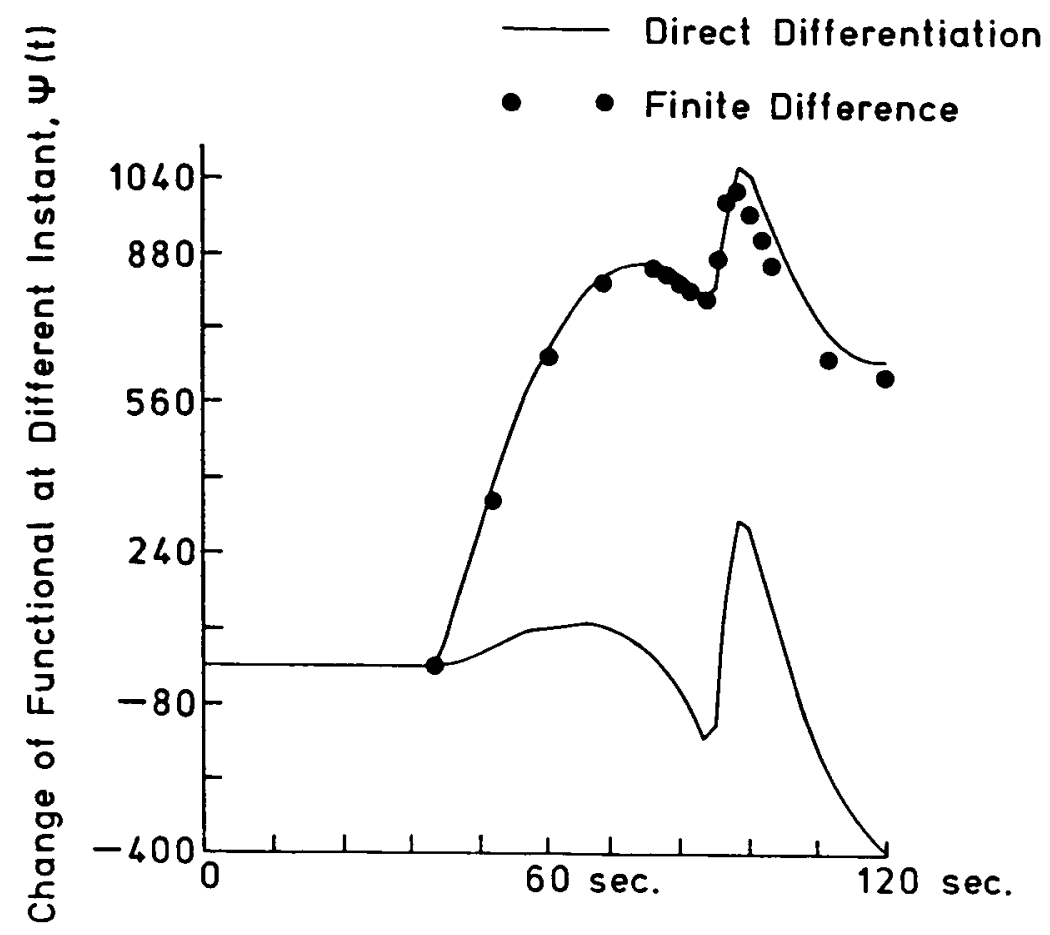

Time

Figure 5. Thermal design derivatives for compressive molding with a jump in $\dot{\mathrm{T}}_{\mathrm{c}}$.

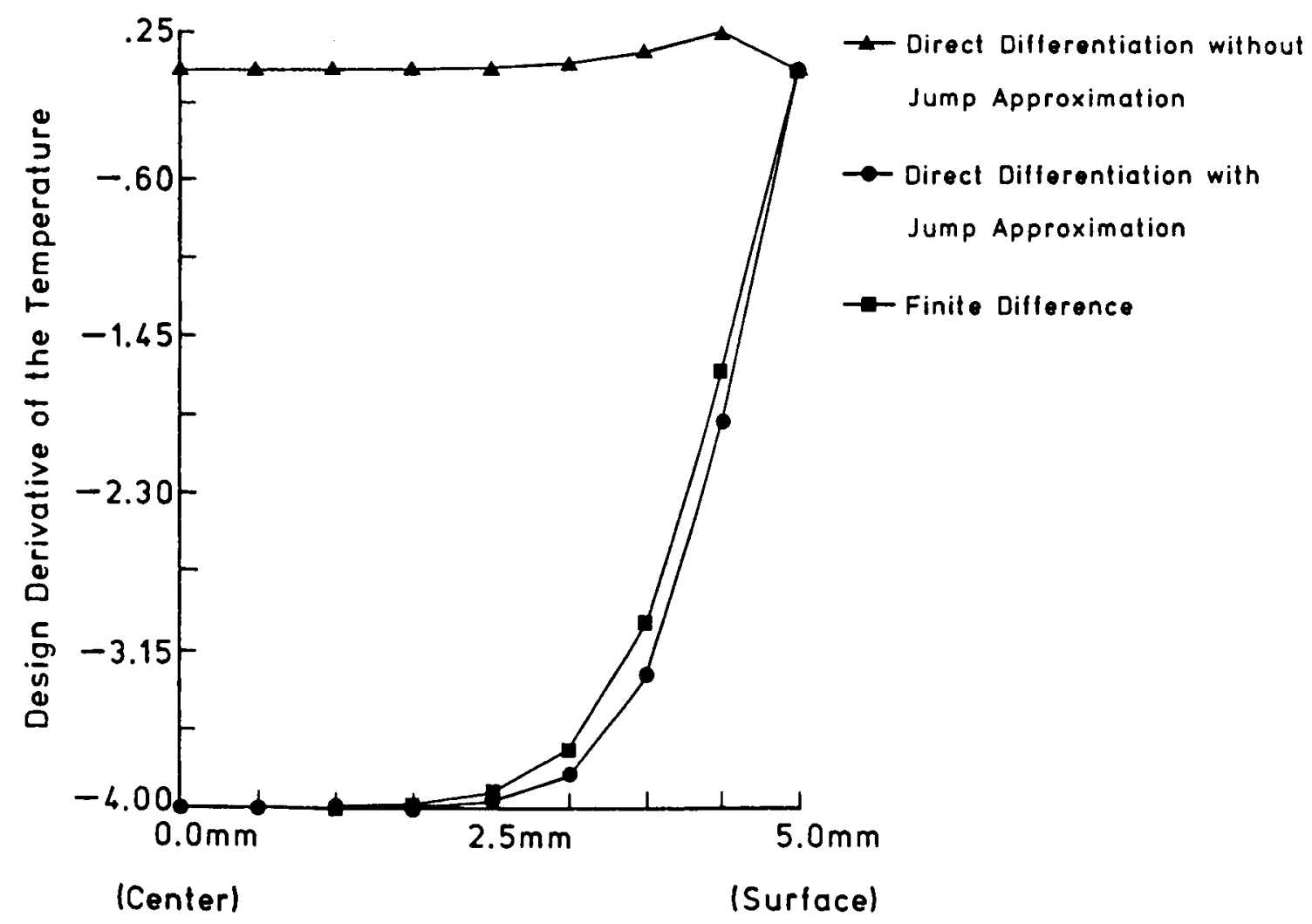

Figure 6. Design derivatives of temperature at time equal to $42 \mathrm{sec}$. 


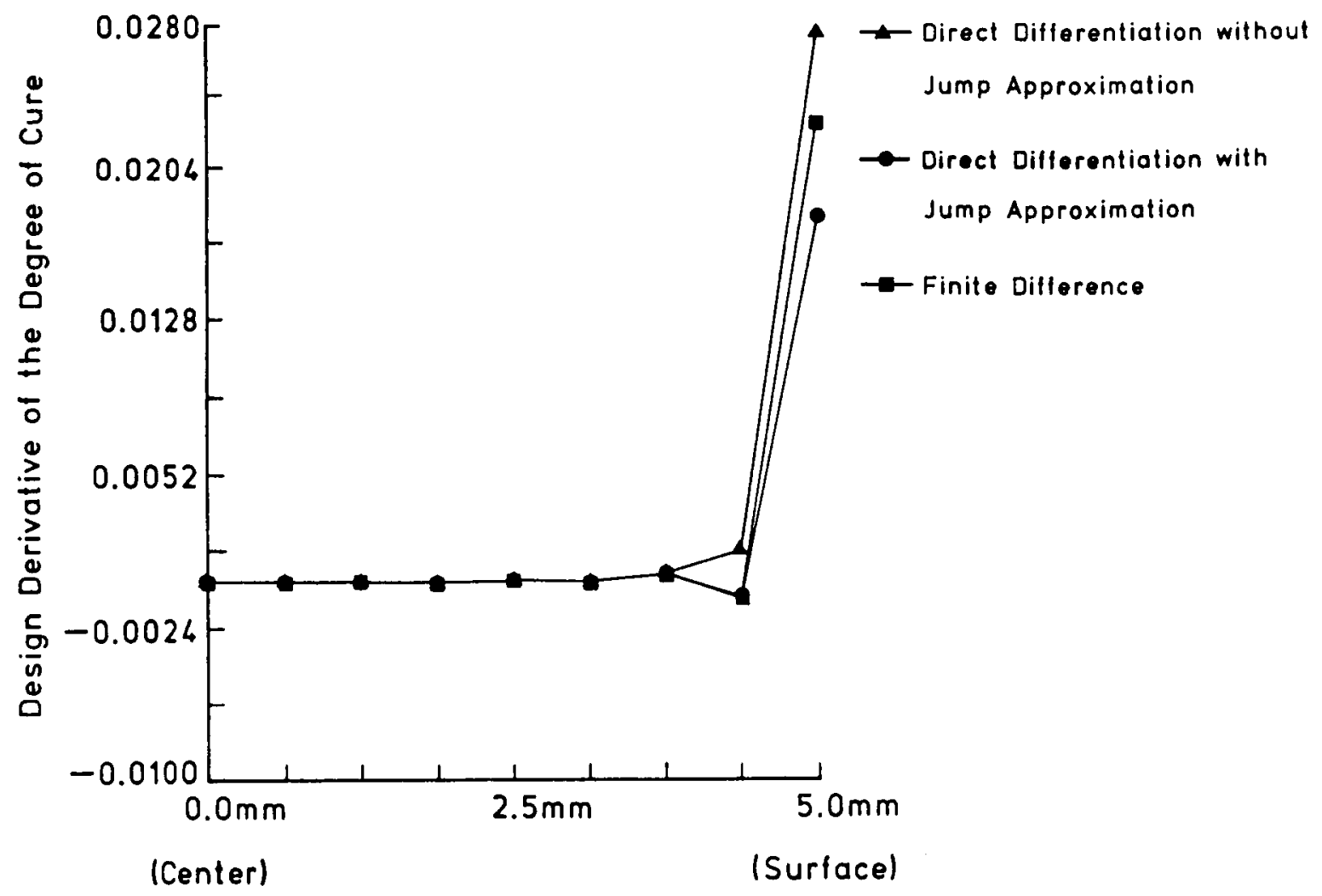

Figure 7. Design derivative of degree of cure at time equal to $42 \mathrm{sec}$.

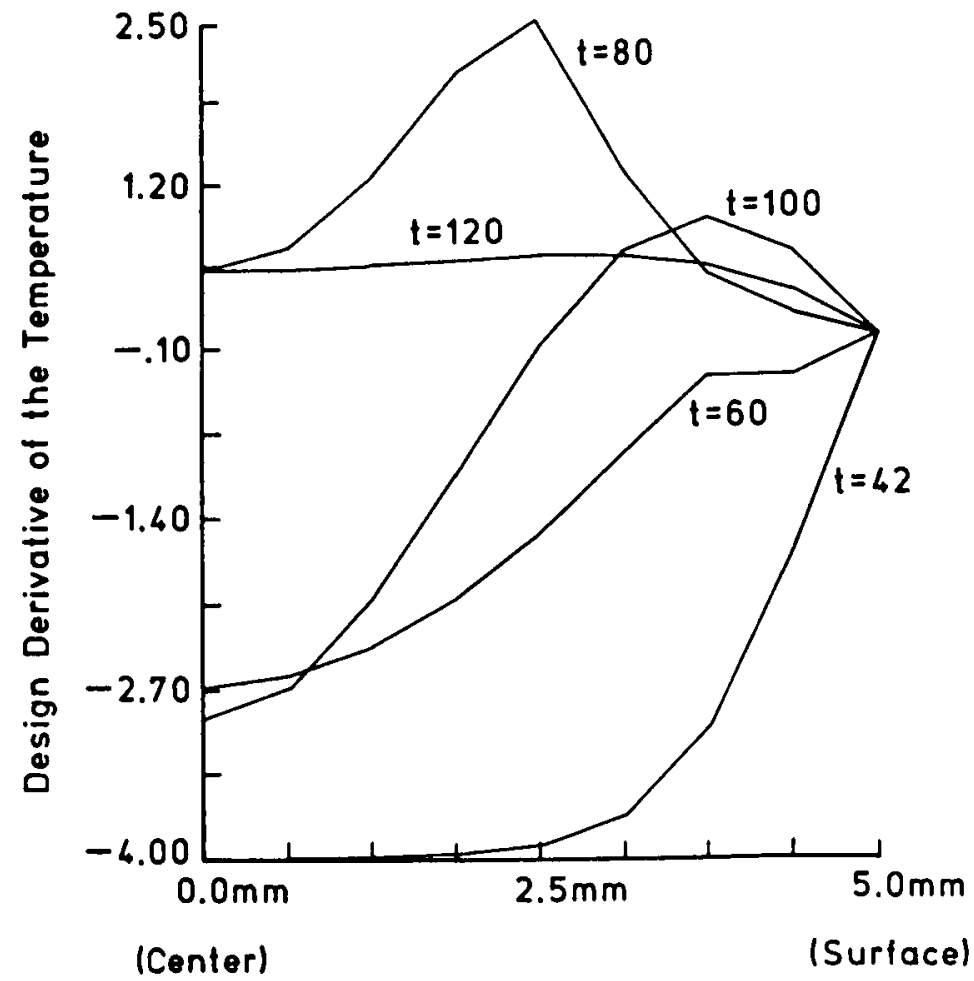

Figure 8. Distribution of $\mathrm{T}^{\prime}$ at different time instants. 


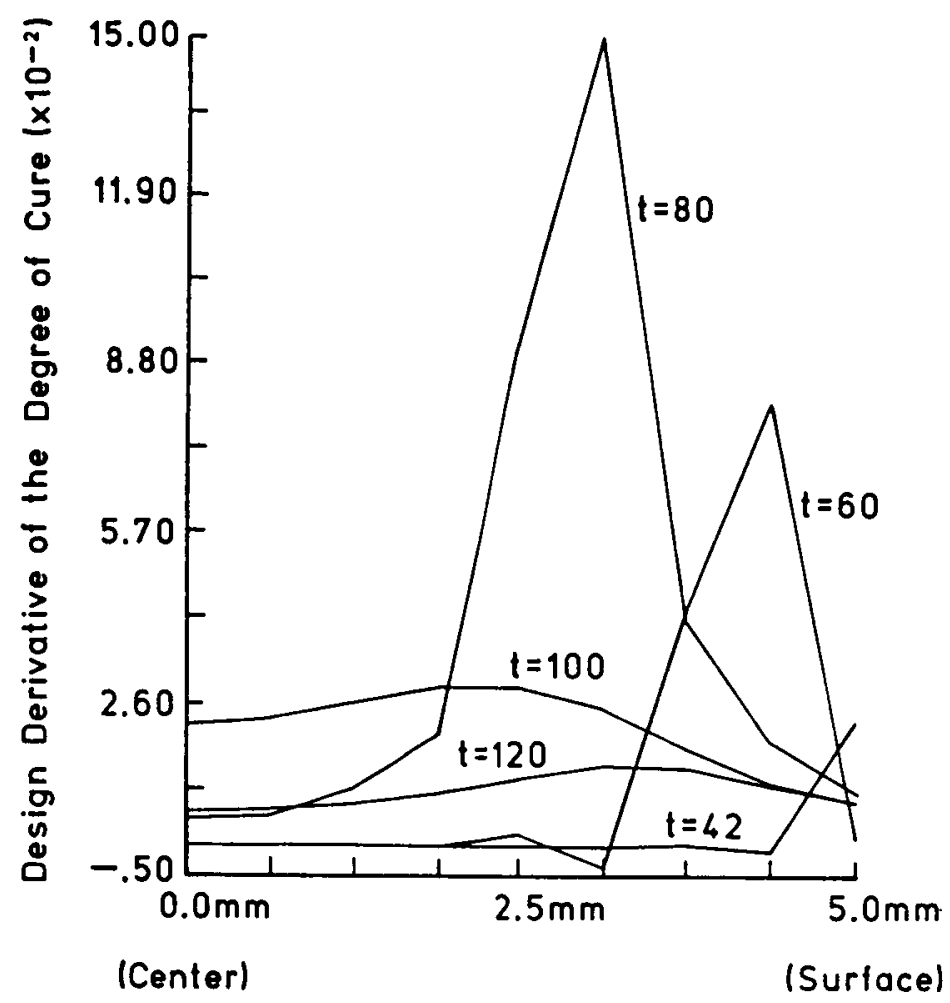

Figure 9. Distribution of $\alpha^{\prime}$ at different time instants.

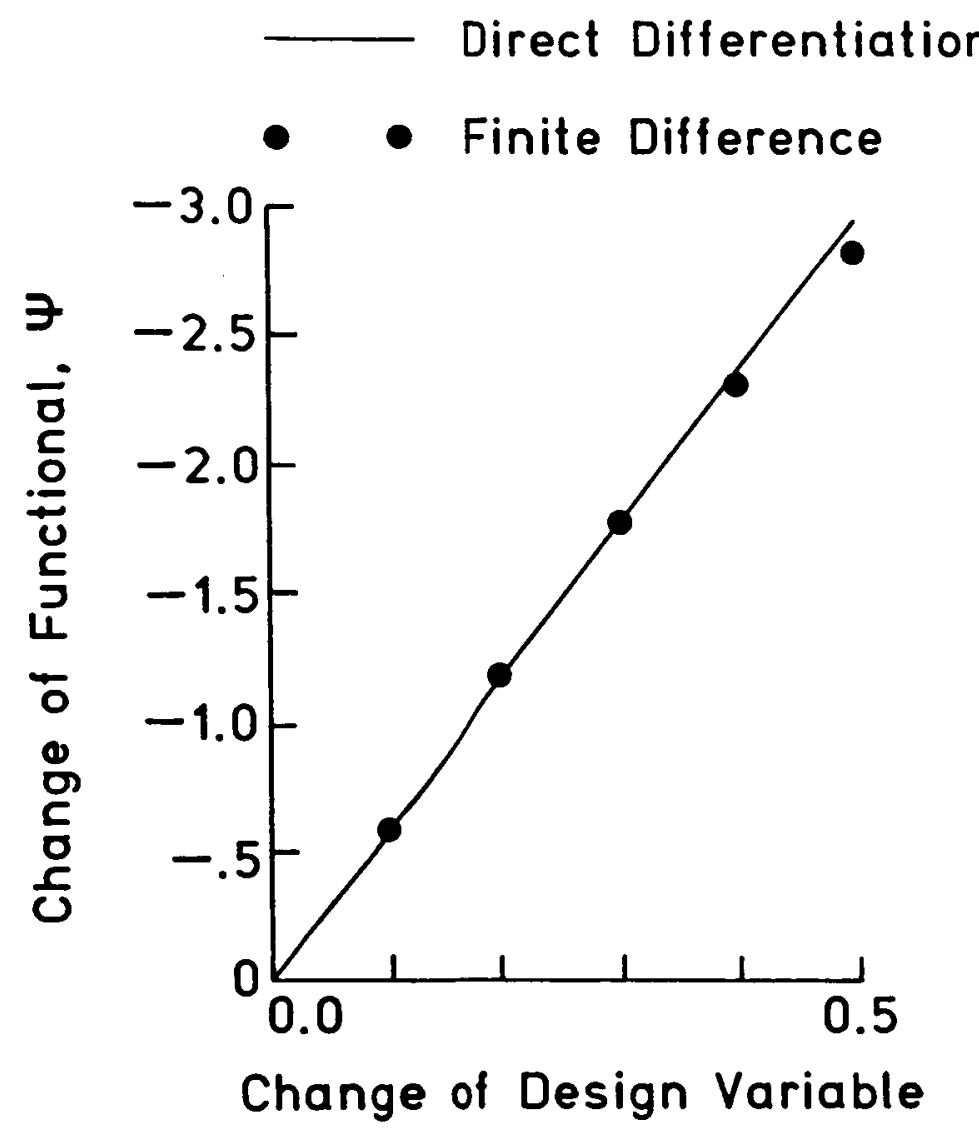

Figure 10. Thermal design derivatives for press molding with a jump in $T_{c}$. 